TITLE:

\title{
Thermal expansion behavior of ultrathin polymer films supported on silicon substrate
}

$\operatorname{AUTHOR}(S)$ :

Miyazaki, T; Nishida, K; Kanaya, T

CITATION:

Miyazaki, T ...[et al]. Thermal expansion behavior of ultrathin polymer films supported on silicon substrate. Physical Review E 2004, 69(6):

061803.

ISSUE DATE:

2004-06

URL:

http://hdl.handle.net/2433/39908

RIGHT:

Copyright 2004 American Physical Society 
PHYSICAL REVIEW E 69, 061803 (2004)

\title{
Thermal expansion behavior of ultrathin polymer films supported on silicon substrate
}

\author{
Tsukasa Miyazaki, ${ }^{1,2, *}$ Koji Nishida, ${ }^{1}$ and Toshiji Kanaya ${ }^{1, \dagger}$ \\ ${ }^{1}$ Institute for Chemical Research, Kyoto University, Uji, Kyoto 611-0011, Japan \\ ${ }^{2}$ Nitto Denko Corporation, 1-1-2, Shimohozumi, Ibaraki, Osaka 567-8680, Japan
}

(Received 16 November 2003; revised manuscript received 23 February 2004; published 22 June 2004)

\begin{abstract}
The thermal expansion behavior of polystyrene (PS) thin films was investigated using x-ray reflectivity, focusing on ultrathin films below $10 \mathrm{~nm}$. It was found that the glass transition temperature $T_{\mathrm{g}}$ decreases with thickness as reported by many researchers while it is almost independent of thickness and constant at $354 \mathrm{~K}$ for films below $\sim 10 \mathrm{~nm}$. The thickness dependence of $T_{\mathrm{g}}$ was well reproduced by a two-layer model consisting of a mobile surface layer with $T_{\mathrm{g}}$ of $354.5 \mathrm{~K}$ and a bulklike layer with $T_{\mathrm{g}}$ of $373 \mathrm{~K}$ (=bulk $T_{g}$ ), suggesting that the so-called immobile dead layer near the substrate is negligible or very thin in this system. This surface $T_{\mathrm{g}}$ of $354 \mathrm{~K}$ was confirmed by the relaxation of surface roughness of as-deposited films at about $354 \mathrm{~K}$. It was also found that the thermal expansivity decreases with thickness in the glassy state as well as in the molten state while the reduction is smaller in the molten state.
\end{abstract}

DOI: 10.1103/PhysRevE.69.061803

PACS number(s): 36.20.-r, 64.70.Pf, 65.60.+a, 68.60.Dv

\section{INTRODUCTION}

Many studies have been performed on the structure and dynamics of amorphous materials near the glass transition temperature $T_{\mathrm{g}}$ using various experimental techniques to understand the nature of glass-forming materials [1-3]. About ten years ago, it was found that the $T_{\mathrm{g}}$ of thin polystyrene films decreases below the bulk $T_{\mathrm{g}}$, depending on thickness $[4,5]$. Since then, the glass transition of thin polymer films has been extensively studied [6]. One of the objectives of these studies is to elucidate the characteristic length scale responsible for the glass transition, which increases as the temperature is lowered towards $T_{\mathrm{g}}[7,8]$. In confinement systems, the correlation length is truncated by the dimension of the restrictive geometry when it reaches the system size, giving information about the characteristic length scale without knowing any physical nature of the correlations. After the first report on the $T_{\mathrm{g}}$ reduction in polystyrene (PS) thin films supported on silicon by Keddie et al. [4,5], similar experimental results have been reported by many researchers $[9,10]$. Furthermore, Brillouin light scattering studies by Forrest $e t$ al. $[11,12]$ elucidated that freely standing PS thin films show much larger $T_{\mathrm{g}}$ reductions than supported films and there is a threshold thickness for the $T_{\mathrm{g}}$ reduction, depending on molecular weight.

In some reports $[13,14]$, the $T_{\mathrm{g}}$ reduction was discussed in relation to the characteristic length scale responsible for the glass transition. However, such a $T_{\mathrm{g}}$ reduction is observed only for polymer films having negligible or very weak interactions with substrates like PS and Si wafers. In fact, the glass transition temperature $T_{\mathrm{g}}$ increases with decreasing film thickness for poly(methyl methacrylate) (PMMA) [4,5], which has strong interactions with $\mathrm{Si}$ substrate. It is now

\footnotetext{
*Corresponding author. Electronic address: tsukasa_miyazaki@gg.nitto.co.jp

†Corresponding author. kanaya@scl.kyoto-u.ac.jp
}

obvious that pure finite-size effects are hardly extracted from these experiments for thin polymer films supported on a solid substrate because surface and interface effects on $T_{\mathrm{g}}$ are not negligible [4,5,15-17]. Hence, before discussing finite-size effects precisely, it is necessary to clarify the surface and interface effects.

Regarding the thickness dependence of thermal expansivity in glassy and molten states there are still contradictory experimental results, depending on the experimental techniques $[4,10,18,19]$ and thermal history of the thin films. [20-22] In previous papers [21,22], we have investigated annealing effects on the thickness of polystyrene thin films to find that the negative expansivity in the glassy state reported by Orts et al. [20] is partly caused by the unrelaxed structure due to a lack of annealing. However, it is not all of the origins for the apparent negative expansivity. In other words, the zero or very small expansivity in the glassy state is inherent in very thin films.

In this article, we have investigated the glass transition temperature $T_{\mathrm{g}}$ and the thermal expansivity of PS thin films using x-ray reflectivity (XR), focusing on ultrathin films below $10 \mathrm{~nm}$. The thickness of the surface mobile layer and/or the immobile dead layer near substrate is considered to be below $10 \mathrm{~nm}[4,10,18,19]$, and hence a detailed characterization of the ultrathin films must throw light on the characteristic nature. XR is a suitable method for this purpose rather than other methods like ellipsometry [23] because of its high thickness resolution of $\sim 0.01 \mathrm{~nm}$, giving very precise values of thermal expansivity.

\section{EXPERIMENT}

In this study, we used two polystyrenes with molecular weights $M_{\mathrm{w}}=3.03 \times 10^{5}$ (Polymer Source Inc.) $\left(\mathrm{PS}_{303 \mathrm{k}}\right)$ and $M_{\mathrm{w}}=2.89 \times 10^{6}$ (Toso Inc.) $\left(\mathrm{PS}_{2890 \mathrm{k}}\right)$. The molecular weight distributions of both PS's are $M_{\mathrm{w}} / M_{\mathrm{n}}=1.09$, where $M_{\mathrm{w}}$ and $M_{\mathrm{n}}$ are the weight average and the number average of the molecular weight, respectively. 
Polystyrene thin films for XR measurements were prepared on cleaned $\mathrm{Si}(111)$ wafers. Silicon wafers prior to deposition were rinsed in ethanol and then in distilled water. Furthermore, dried wafers were cleaned in the UV ozone cleaner (NL-UV253, Nippon Laser \& Electronics Lab.). Polymer solutions with various concentrations were filtered ( $2 \mu \mathrm{m}$ pore size) and spun cast at $2000 \mathrm{rpm}$ on cleaned silicon wafers. The thickness of the polymer film was controlled by varying the polymer concentration in solution.

XR measurements were performed using a homebuilt $\mathrm{X}$-ray reflectometer which was based on a conventional powder diffractometer. Refer to Refs. [24,25] for the data analysis in this study. The sample environment was maintained in a chamber with beryllium windows under a vacuum. The chamber was placed on the $\theta$ axis of the reflectometer. The sample temperature during the measurements was controlled within $\pm 0.1 \mathrm{~K}$ with a temperature controller.

As-deposited PS thin films were introduced in the chamber and kept for $1 \mathrm{~h}$ under vacuum. XR measurements for as-deposited PS thin films were performed by every $5 \mathrm{~K}$ from $298 \mathrm{~K}$ to $423 \mathrm{~K}$ to observe the thermal relaxation behavior. The samples were kept at $423 \mathrm{~K}$ for $38 \mathrm{~h}$ in total in vacuum after this first heating ramp and then cooled down to $298 \mathrm{~K}$. XR measurements were again performed by every $5 \mathrm{~K}$ from $298 \mathrm{~K}$ to $423 \mathrm{~K}$ to determine the thermal expansivities in the glass and the melt as well as the glass transition temperature $T_{\mathrm{g}}$ from the change of the thermal expansivities. As reported in the previous paper [22], after annealing at $423 \mathrm{~K}$ for more than $\sim 2 \mathrm{~h}$, the thermal expansivity and the glass transition temperature $T_{\mathrm{g}}$ are not affected by further annealing.

It took about $10 \mathrm{~min}$ for one XR measurement at a given temperature and the heating rate was $1 \mathrm{~K} / \mathrm{min}$, meaning that one $T_{\mathrm{g}}$ and thermal expansivity determination took about $7 \mathrm{~h}$. The samples were not exposed to air after they were introduced in the chamber of the reflectometer. A $\theta$ rocking scan was performed by every $10 \mathrm{~K}$ during the measurements to check the sample alignment. Note that dewetting was observed in the films below $5 \mathrm{~nm}$ for $\mathrm{PS}_{303 \mathrm{k}}$ and below $7 \mathrm{~nm}$ for $\mathrm{PS}_{2890 \mathrm{k}}$ above $\sim 400 \mathrm{~K}$ and hence such film data were not included in this report.

\section{RESULTS AND DISCUSSION}

Figure 1 shows the observed reflectivity profiles for the thinnest $\mathrm{PS}_{303 \mathrm{k}}$ film with initial thickness of $6.3 \mathrm{~nm}$ at various temperatures. The solid curves are the results of fits, agreeing with the observed data very well. From the fits we have evaluated the thickness, the surface and interfacial roughness, and the density. Figure 2 shows a typical example of the temperature dependence of the thickness of $\mathrm{PS}_{303 \mathrm{k}}$ thin films with various initial values. The thicknesses were normalized to that at $298 \mathrm{~K}$ for each sample. For all samples, a discontinuous change of the thermal expansivity was clearly observed, showing a definite glass transition temperature $T_{\mathrm{g}}$, which is indicated by an arrow in the figure. The expansivities in the glassy and molten states, which are shown by dashed and solid lines in the figure, respectively, are also definitely determined in the measurements. The temperature

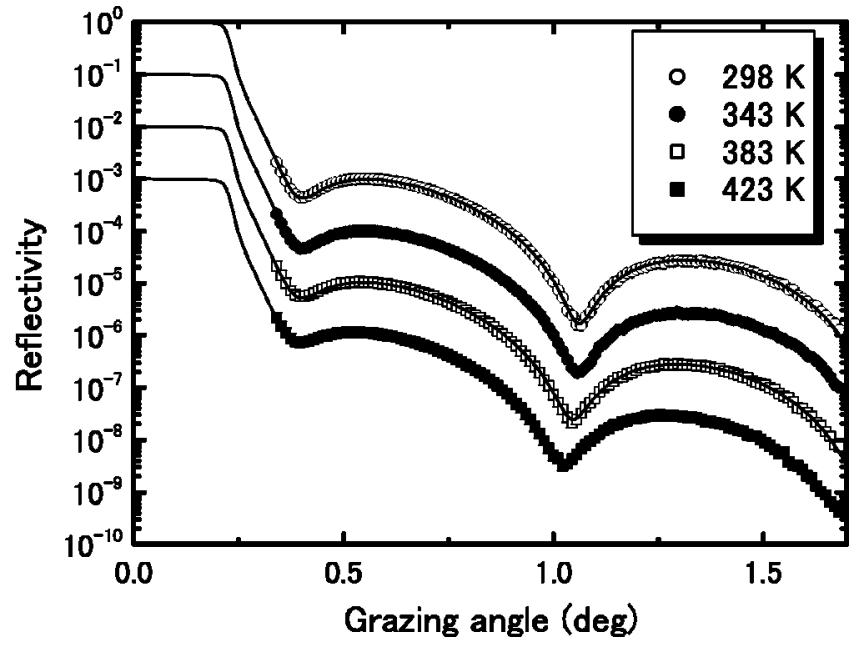

FIG. 1. X-ray reflectivity profiles for $\mathrm{PS}_{303 \mathrm{k}}$ thin film supported on $\mathrm{Si}$ substrate at various temperatures after annealing at $150^{\circ} \mathrm{C}$ for $38 \mathrm{~h}$. Initial thickness at $298 \mathrm{~K}$ is $6.3 \mathrm{~nm}$.

dependence of the thickness was compared with that of the density for the $\mathrm{PS}_{303 \mathrm{k}}$ film with initial thickness of $6.3 \mathrm{~nm}$ in Fig. 3 where the data were normalized to the lowest values for comparison. The correlation between two quantities is very good, suggesting that the expansion of the film normal to the surface direction is caused by the density change.

Before going into a detailed discussion of the results, we would like to mention the annealing time effects on the glass transition temperature $T_{\mathrm{g}}$ and the thermal expansivity. In this work, we annealed the samples at $150^{\circ} \mathrm{C}$ for $38 \mathrm{~h}$. This annealing time is longer than the terminal time for the sample with $M_{\mathrm{w}}=303 \mathrm{k}(\sim 2.5 \mathrm{~min})$, but not for $M_{\mathrm{w}}=2890 \mathrm{k}$ $(\sim 90 \mathrm{~h})$ [26]. However, according to the discussion of Dalnoki-Veress et al. [27] an annealing time longer than the segmental relaxation (Rouse relaxation) is enough for the determination of $T_{\mathrm{g}}$. Our annealing time $\left(38 \mathrm{~h}\right.$ at $\left.150^{\circ} \mathrm{C}\right)$ is much longer than the Rouse time $\left(\sim 0.01 \mathrm{~s}\right.$ at $\left.150^{\circ} \mathrm{C}\right)$ [26].

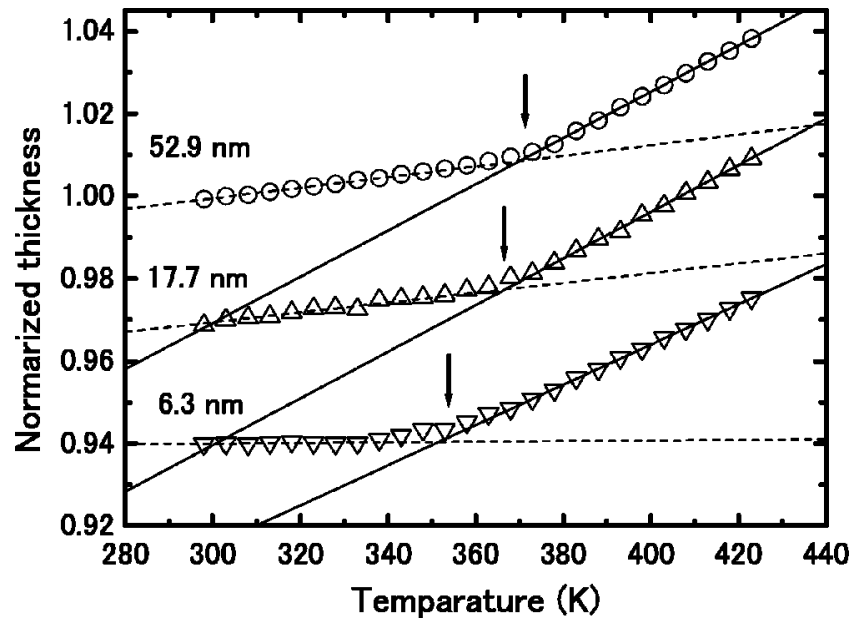

FIG. 2. Temperature dependence of film thicknesses for $\mathrm{PS}_{303 \mathrm{k}}$ supported on $\mathrm{Si}$ substrate. The data are normalized to the value at $298 \mathrm{~K}$ and vertically offset for clarify. Initial thickness at $298 \mathrm{~K}$ is $52.9 \mathrm{~nm}(\bigcirc), 17.7 \mathrm{~nm}(\Delta)$, and $6.3 \mathrm{~nm}(\nabla)$. 


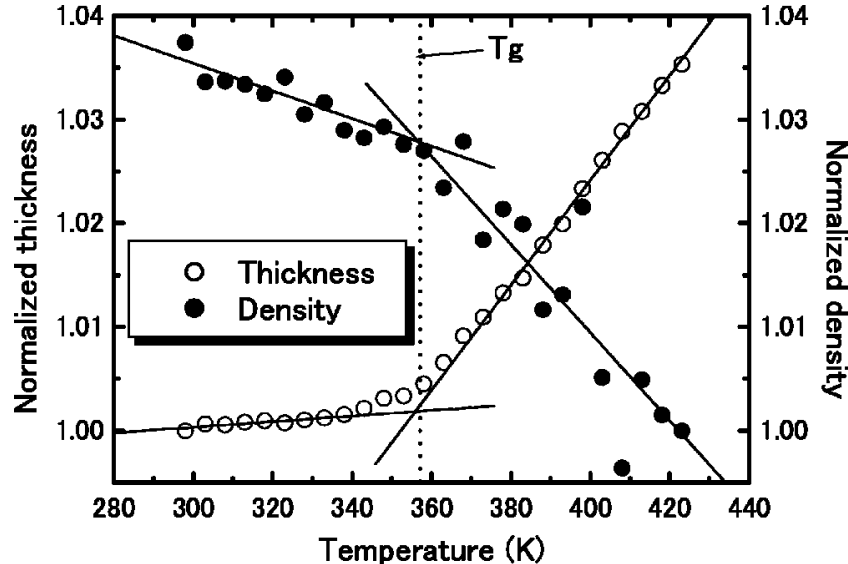

FIG. 3. Comparison of the temperature dependence between the thickness and the density for $\mathrm{PS}_{303 \mathrm{k}}$ thin film with initial thickness of $6.3 \mathrm{~nm}$. Both the thickness and the density are normalized to the lowest values.

Experimentally, we also examined the annealing time effects on the film thickness in the previous paper [22] and found two relaxation (contraction) processes during the annealing at $150{ }^{\circ} \mathrm{C}$. One is a fast structural relaxation process completed within $\sim 2 \mathrm{~h}$, and the other is an extremely slow one with relaxation time of 30-50 h, especially for thin films below $\sim 20 \mathrm{~nm}$. It was experimentally found that after the annealing at $150^{\circ} \mathrm{C}$ for $2 \mathrm{~h}$ the glass transition temperature $T_{\mathrm{g}}$ and the thermal expansivity are not affected by the further annealing if the time to measure them is shorter than the relaxation time of the slow contraction process. All the measurements in this work satisfied these annealing conditions except those on the as-deposited films. Exactly speaking, the values of expansivity reported here are not equilibrium ones because they are slightly affected by the extremely slow relaxation process [22] as well as the reptation process for $M_{\mathrm{w}}=2890 \mathrm{k}$ in measurements longer than the relaxation times.

Figure 4 shows the thickness dependence of the $T_{\mathrm{g}}$ for $\mathrm{PS}_{303 \mathrm{k}}$ and $\mathrm{PS}_{2890 \mathrm{k}}$ thin films. The glass transition temperature $T_{\mathrm{g}}$ of $\mathrm{PS}_{303 \mathrm{k}}$ thin film begins to decrease with thickness at $\sim 40 \mathrm{~nm}$ as reported by many groups [28]. The $T_{\mathrm{g}}$ of $\mathrm{PS}_{2890 \mathrm{k}}$ also decreases with thickness while the reduction in $T_{\mathrm{g}}$ seems larger than that of $\mathrm{PS}_{303 \mathrm{k}}$, suggesting that the $T_{\mathrm{g}}$ of the supported thin films depends on molecular weight. It is well known that the glass transition temperature $T_{\mathrm{g}}$ in bulk does not depend on the molecular weight in the highmolecular-weight range, roughly speaking above $M_{\mathrm{w}}$ $=10000$ for PS, while it increases with molecular weight according to $T_{\mathrm{g}}=T_{\mathrm{g}}^{\infty}-K / M_{W}$ in the low-molecular-weight range [29], where $T_{\mathrm{g}}^{\infty}$ and $K$ are $T_{\mathrm{g}}$ at infinite $M_{\mathrm{w}}$ and a constant. Hence, it was a great surprise when the molecular weight dependence of $T_{\mathrm{g}}$ was reported in freely standing films of high-molecular-weight PS [27,30]. There have been reported some $T_{\mathrm{g}}$ data for supported thin films with various molecular weights, but the molecular weight dependence of $T_{\mathrm{g}}$ has not been explicitly discussed except for the Fukao and Miyamoto's dielectric data [31] as far as we know. This may be because of the rather small number of points in each molecular weight data subset coupled with the substantial data

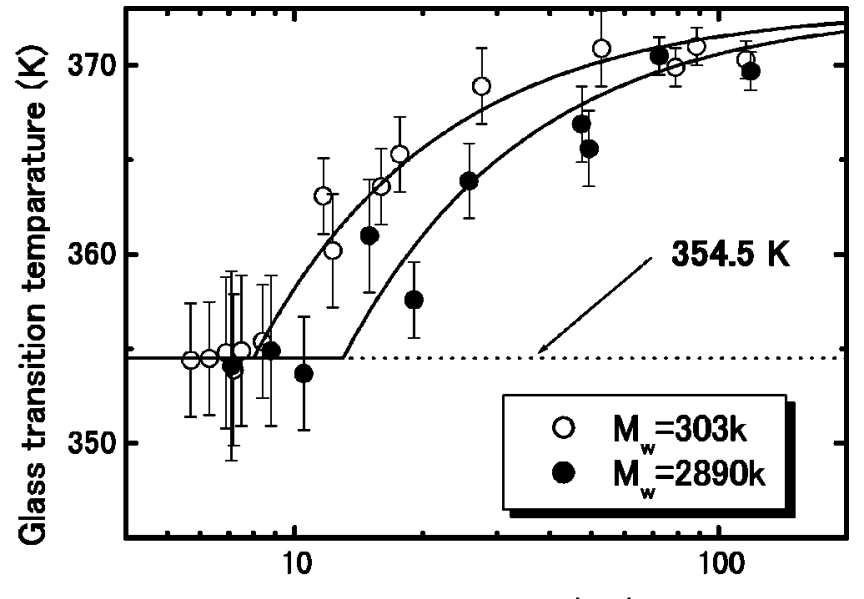

Film thickness (nm)

FIG. 4. Thickness dependence of $T_{\mathrm{g}}$ for PS thin films supported on Si substrate for $M_{\mathrm{w}}=303 \mathrm{k}(\bigcirc)$ and $M_{\mathrm{w}}=2890 \mathrm{k}(\bullet)$. Solid curves are the results of fits with Eq. (2) (see text). The parameters obtained in the fits are $T_{\mathrm{g}}^{\text {bulk }}=373 \mathrm{~K}, T_{\mathrm{g}}^{\text {surf }}=354.5 \mathrm{~K}$, and $A=8 \mathrm{~nm}$ for PS thin films with $M_{\mathrm{w}}=303 \mathrm{k}$ and $A=13 \mathrm{~nm}$ for PS thin films with $M_{\mathrm{w}}=2890 \mathrm{k}$.

scatter in the reported data [9]. The present observation suggests that the $T_{\mathrm{g}}$ of supported thin films also depends on the molecular weight, although the effect is not so large compared with freely standing films. In order to explain the molecular weight dependence of $T_{\mathrm{g}}$ for freely standing thin films, a "sliding motion" mechanism was proposed by de Gennes [32]. In this model, a chain advances along its own path. The free volume required for the sliding motion involves only the side chains and is much less than bulk cooperative motion. Sliding is blocked in the bulk because chain ends would have to invade new territory, and this requires a large free volume. Near a free surface, the situation could be different: the monomers in direct contact with the air are nearly fluid and sliding motion easily occurs near a free surface. Such sliding motion may be a possible explanation for the molecular weight dependence of $T_{\mathrm{g}}$ in supported thin films.

Another interesting feature in Fig. 4 is that the glass transition temperature $T_{\mathrm{g}}$ is almost independent of thickness and constant $(\sim 355 \mathrm{~K})$ in the range below about $10 \mathrm{~nm}$ for both $\mathrm{PS}_{303 \mathrm{k}}$ and $\mathrm{PS}_{2890 \mathrm{k}}$. This is not the first report on $T_{\mathrm{g}}$ for thin films less than $10 \mathrm{~nm}$. In the literature, in fact, we can find some $T_{\mathrm{g}}$ data below $10 \mathrm{~nm}$ [28]. However, the data points are too much scattered in this range, depending on experimental methods and thermal history of samples, to conclude the thickness dependence. Taking into account that XR has a high thickness resolution compared with other methods like ellipsometry [23] and the present thin films are well annealed, we believe that the glass transition temperature $T_{\mathrm{g}}$ is almost constant for ultrathin films below $\sim 10 \mathrm{~nm}$.

It is very natural to consider a mobile surface layer with lower $T_{\mathrm{g}}^{\text {surf }}$ than the bulk $T_{\mathrm{g}}^{\text {bulk }}$ on the surface of the thin film as many experiments suggested. Then, we adopted a twolayer model. Assuming that the surface and bulklike layers are $A$ and $D-A$ in thickness and have glass transition temperatures $T_{\mathrm{g}}^{\text {surf }}$ and $T_{\mathrm{g}}^{\text {bulk }}\left(T_{\mathrm{g}}^{\text {surf }}<T_{\mathrm{g}}^{\text {bulk }}\right)$, respectively, where 


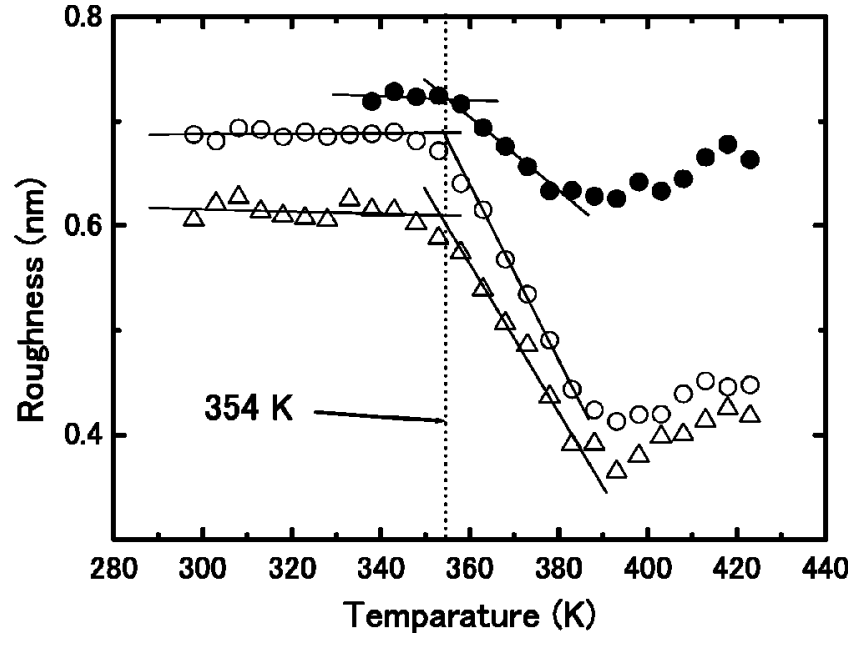

FIG. 5. Temperature dependence of the surface roughness for the as-deposited PS thin films. PS with $M_{\mathrm{w}}=303 \mathrm{k}$ and thickness of $16 \mathrm{~nm}(\bigcirc)$ and $13 \mathrm{~nm}(\Delta)$, and PS with $M_{\mathrm{w}}=2980 \mathrm{k}$ and thickness of $25.8 \mathrm{~nm}(\bullet)$. Surface roughness begins to relax at $354 \mathrm{~K}$ irrespective of the glass transition temperature of the films. For $T_{\mathrm{g}}$ of the films, see Fig. 4.

$D$ is the total thickness of the film, the thickness dependence of the apparent $T_{\mathrm{g}}$ is given as follows:

$$
\begin{aligned}
T_{\mathrm{g}} & =\frac{1}{D}\left(A T_{g}^{\text {surf }}+(D-A) T_{g}^{\text {bulk }}\right) \text { for } D \geqslant A \\
& =T_{g}^{\text {surf }} \text { for } D<A .
\end{aligned}
$$

Equation (1) was fitted to the observed data and the results of the fits are shown in Fig. 4 by solid lines. The fitness is good. The parameters obtained in the fits are $T_{g}^{b u l k}=373 \mathrm{~K}, T_{g}^{\text {surf }}$ $=354.5 \mathrm{~K}$ for both $\mathrm{PS}_{303 \mathrm{k}}$ and $\mathrm{PS}_{2890 \mathrm{k}}$, and $A=8 \mathrm{~nm}$ for $\mathrm{PS}_{303 \mathrm{k}}$ and $A=13 \mathrm{~nm}$ for $\mathrm{PS}_{2890 \mathrm{k}}$, respectively. The two-layer model suggests that the dead layer near the substrate is negligible or very thin in contrast to some previous works $[18,19]$.

The surface $T_{\mathrm{g}}$ and the surface layer thickness of PS thin films have been studied using scanning microscopy by some researchers. Fischer has studied the surface of bulk PS with $M_{\mathrm{w}}=276 \mathrm{k}$ using thermal probe atomic force microscopy (AFM) [33] and found that the surface layer is about $14 \mathrm{~nm}$ thick and the surface $T_{\mathrm{g}}$ is $356 \mathrm{~K}$. Scanning viscoelasticity microscopy was also used to study the surface region of the PS films in a molecular weight range of $M_{\mathrm{w}}=4.9-1450 \mathrm{k}$ and it was found that the surface $T_{\mathrm{g}}$ is always lower than the bulk $T_{\mathrm{g}}$ while it depends on the molecular weight. The surface $T_{\mathrm{g}}$ of PS with $M_{\mathrm{n}}=250 \mathrm{k}$ is about $350 \mathrm{~K}$ while that of PS with $M_{\mathrm{n}}=1450 \mathrm{k}$ is about $360 \mathrm{~K}$ [34]. The thickness of the surface region was also evaluated by the same group for PS with $M_{\mathrm{n}}=29 \mathrm{k}$ using dynamic secondary-ion-mass spectroscopy to be about $4.8 \mathrm{~nm}$ [35]. These reported data are not completely in agreement, but it is safely mentioned that the surface $T_{\mathrm{g}}$ is about $10-30 \mathrm{~K}$ lower than the bulk $T_{\mathrm{g}}$ and the surface layer is $5-15 \mathrm{~nm}$ thick, being consistent with the present result except very-low-molecular-weight PS.
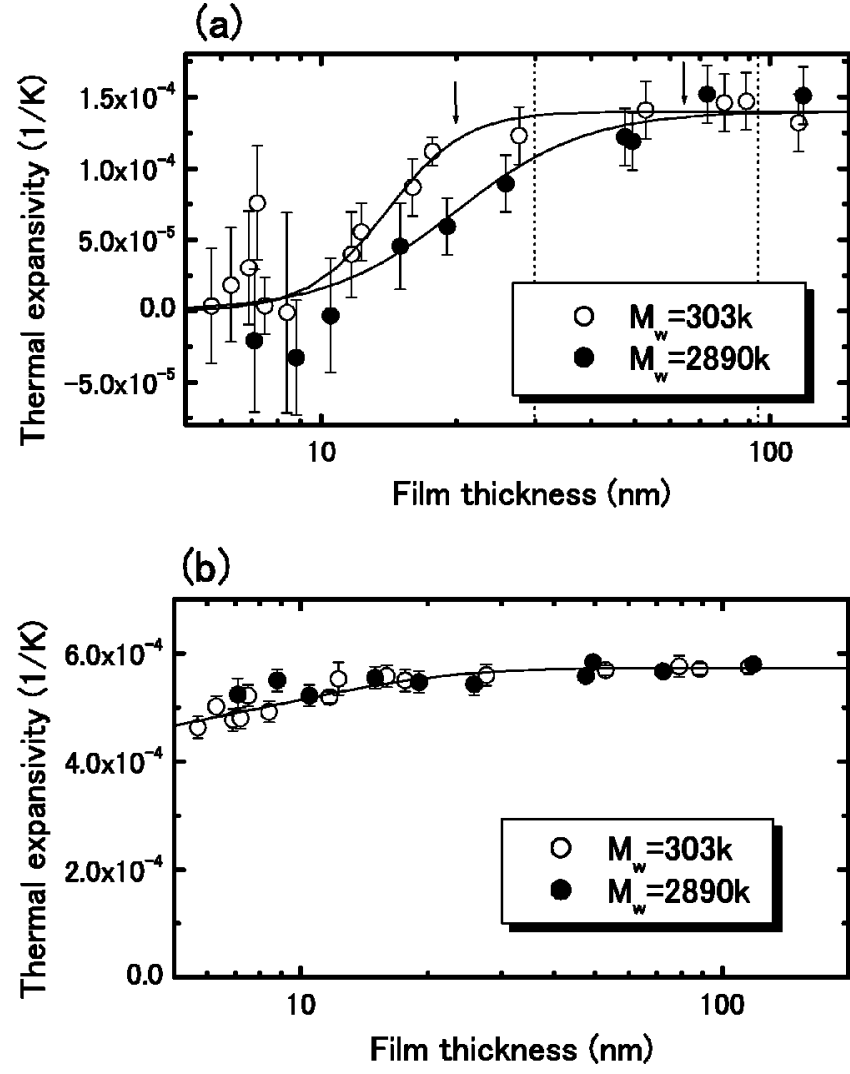

FIG. 6. Thickness dependence of the thermal expansivity of PS thin films (a) in the glassy state and (b) in the molten state. $M_{\mathrm{w}}$ $=303 \mathrm{~K}(\bigcirc), M_{\mathrm{w}}=2890 \mathrm{~K}(\bullet)$. In (a), dashed lines show twice of the radius of gyration, $2 R_{\mathrm{g}}$, and down arrows show the onsets of the reduction in the expansivities.

Fischer's work mentioned above is suggestive. In the work, he showed that the surface roughness could be smoothed with a scanning of the thermal probe above the surface $T_{\mathrm{g}}(=356 \mathrm{~K})$. This suggests that the rough surface of as-deposited films could be relaxed above the surface $T_{\mathrm{g}}$. Hence, we decided to observe surface relaxation behavior of as-deposited PS thin films with a relative rough surface. Figure 5 shows the temperature dependence of the surface roughness for the as-deposited $\mathrm{PS}_{303 \mathrm{k}}$ and $\mathrm{PS}_{2890 \mathrm{k}}$ films with various thicknesses. As temperature increases from room temperature, the surface roughness of the as-deposited films is almost independent of temperature and abruptly begins to decrease at around $354 \mathrm{~K}$, suggesting that the onset temperature of surface relaxation is $354 \mathrm{~K}$ and almost independent of molecular weight. This observation strongly supports the conjecture that the surface $T_{\mathrm{g}}$ of the PS films is about $354 \mathrm{~K}$.

In the next step, we will consider the thickness dependence of thermal expansivity of PS thin films. Thermal expansivities below and above the glass transition temperature $T_{\mathrm{g}}$ were evaluated from the slopes of the straight lines in Fig. 2 and plotted as a function of the thickness in Figs. 6(a) and 6(b), respectively, for both $\mathrm{PS}_{303 \mathrm{k}}$ and $\mathrm{PS}_{2890 \mathrm{k}}$. First, we will focus on the thickness dependence of the thermal expansivity in the glassy state. As seen in Fig. 6(a), the expansivity in the thickness range above $\sim 65 \mathrm{~nm}$ is $\sim 1.3 \times 10^{-4}$ for both $\mathrm{PS}_{303 \mathrm{k}}$ and $\mathrm{PS}_{2890 \mathrm{k}}$, which is close to the expected value 
$\left(1.1 \times 10^{-4} \mathrm{~K}^{-1}\right)$ from the bulk assuming that thin films are constrained along the substrate [16]. On the other hand, as the thickness decreases it begins to decrease at $\sim 20 \mathrm{~nm}$ and $\sim 65 \mathrm{~nm}$ for $\mathrm{PS}_{303 \mathrm{k}}$ and $\mathrm{PS}_{2890 \mathrm{k}}$, respectively, which are indicated by down arrows in the figure, and becomes almost zero below $\sim 10 \mathrm{~nm}$. NR measurement by Wu et al. [36] showed that the thermal expansivity of poly(methyl methacrylate) decreases with thickness, although all the data so far reported are not entirely in agreement on the thermal expansivity in the glassy state, depending on experimental methods $[4,10,18,19]$ and thermal history of the samples [20-22]. We believe that it is worth discussing the present results because $\mathrm{XR}$ is one of the most suitable methods for measuring the thickness due to the high thickness resolution and hence the obtained thermal expansivity is reliable.

We know that the unrelaxed structure due to a lack of annealing makes apparent small or negative expansivity for thin films [21,22]. However, the present result is not the case as discussed above. A clue to the problem is found in the molecular weight dependence of the onset thickness of the decrease in the expansivity. As mentioned above, the expansivity begins to decrease at $\sim 20 \mathrm{~nm}$ and $\sim 65 \mathrm{~nm}$ for $\mathrm{PS}_{303 \mathrm{k}}$ and $\mathrm{PS}_{2890 \mathrm{k}}$, respectively. These values are close to twice of the radius of gyration of a chain, $2 R_{\mathrm{g}}$, which are 30 and $94 \mathrm{~nm}$ for $\mathrm{PS}_{303 \mathrm{k}}$ and $\mathrm{PS}_{2890 \mathrm{k}}$, respectively, indicated by the dashed lines in Fig. 6(a). Exactly speaking, the decrease begins at $\sim(2 / 3) \times 2 R_{\mathrm{g}}$. This implies that the decrease in the expansivity is caused by the chain confinement in the thin films.

Recent inelastic neutron scattering experiments on polycarbonate [37] and PS [38] have shown that the mean-square displacement $\left\langle u^{2}\right\rangle$ decreases with film thickness. In the harmonic assumption, $\left\langle u^{2}\right\rangle$ is related to the force constant $f$ through $f=k_{B} T /\left\langle u^{2}\right\rangle$, suggesting that the potential becomes harder as the film decreases in thickness. If the anharmonic contribution in the potential is taken into account, the increase in the force constant may explain the decrease in the thermal expansivity with thickness in the glassy state.

In the melt above $T_{\mathrm{g}}$ the expansivity also decreases with thickness for both $\mathrm{PS}_{303 \mathrm{k}}$ and $\mathrm{PS}_{2890 \mathrm{k}}$ as seen in Fig. 6(b), although the reduction is very small compared with that in the glassy state. From the data in Fig. 6(b) it is hard to say anything about the molecular weight dependence. In some papers $[18,19]$ the reduction of the expansivty in the melt has been reported and explained in terms of a three-layer model including the immobile dead layer (or the glassy layer) near the interface between the film and substrate. As mentioned above, the present analysis of the thickness dependence of $T_{\mathrm{g}}$ by the two-layer model suggests that the dead layer near the interface is negligible or very small in this system, implying that the confinement effect is more plausible for the reduction of the melt expansivity than the effect of the dead layer, at least within the present analysis.

In this study, we have investigated the thickness of PS thin films as a function of temperature using $\mathrm{XR}$, focusing on the ultrathin films below $10 \mathrm{~nm}$. The films were annealed at $423 \mathrm{~K}$ or $50 \mathrm{~K}$ above the bulk $T_{\mathrm{g}}$ for $38 \mathrm{~h}$ to avoid the structure relaxation during the measurements. As reported in some papers, we also found that the $T_{\mathrm{g}}$ decreases with thickness below $\sim 40 \mathrm{~nm}$ while the reduction is larger in the larger molecular weight. What we found in this work is that the $T_{\mathrm{g}}$ is independent of thickness and constant, $354 \mathrm{~K}$, below $\sim 10 \mathrm{~nm}$. Assuming the two-layer model this would indicate that there exists a surface mobile layer $\sim 10 \mathrm{~nm}$ thick with $T_{\mathrm{g}}$ of $354 \mathrm{~K}$, but not the immobile dead layer near the interface between the film and substrate. We also found that the thermal expansivity in the glassy state decreases with thickness below about twice $R_{\mathrm{g}}$. The expansivity in the melt also decreases with thickness but the reduction is smaller than in the glass. The reduction in the expansivity in the glassy state was assigned to the confinement effect because the onset thickness of the reduction is close to the twice the radius of gyration of a chain, $2 R_{\mathrm{g}}$.

\section{ACKNOWLEDGMENTS}

The authors wish to thank Professor K. Fukao and Professor $\mathrm{H}$. Watanabe for their valuable discussions.
[1] Proceedings of Yukawa International Seminar 1996 Kyoto, 1997, (YKIS'96), edited by T. Odagaki, Y. Hiwatari, and J. Matsui [Prog. Theor. Phys. Suppl. 126 (1997)].

[2] Proceedings of The Third International Discussion Meeting on Relaxation in Complex Systems, Vigo, 1997, edited by K. L. Ngai, E. Riande, and M. D. Ingram [J. Non-Cryst. Solids, 235/ 237 (1998)].

[3] Proceedings of Third Workshop on Non-equilibrium Phenomena in Supercoooled Fluids, Glasses and Amorphoous Materials, Pisa, 2002, edited by L. Andreozzi, M. Giordano, D. Leporini, and M. Tosi [J. Phys.: Condens. Matter 26 (2003)].

[4] J. L. Keddie, R. A. Jones, and R. A. Cory, Europhys. Lett. 27, 59 (1994).

[5] J. L. Keddie, R. A. Jones, and R. A. Cory, Faraday Discuss. 98, 219 (1994)

[6] A. Karim and S. Kumar, in Polymer Surfaces, Interfaces and
Thin Films, edited by A. Karim and S. Kumar (World Scientific, Singapore, 2000).

[7] E. Donth, Relaxation and Thermodynamics of Polymers: Glass Transition (Akademie-Verlag, Berlin, 1992).

[8] E. W. Fischer, E. Donth, and W. Steffen, Phys. Rev. Lett. 68, 2344 (1992).

[9] J. A. Forrest and R. A. L. Jones, in Polymer Surfaces, Interfaces and Thin Films, edited by A. Karim and S. Kumar (World Scientific, Singapore, 2000), p. 251.

[10] S. Kawana and R. A. L. Jones, Phys. Rev. E 63, 021501 (2001).

[11] J. A. Forrest, K. Dalnoki-Veress, J. R. Stevens, and J. R. Dutcher, Phys. Rev. Lett. 77, 2002 (1996).

[12] J. A. Forrest, K. Dalnoki-Veress, and J. R. Dutcher, Phys. Rev. E 56, 5705 (1997).

[13] J. A. Forrest and J. Mattsson, Phys. Rev. E 61, R53 (2000). 
[14] J. Mattsson, J. A. Forrest, and L. Borjesson, Phys. Rev. E 62, 5187 (2000).

[15] J. L. Keddie and R. A. L. Jones, Isr. J. Chem. 35, 21 (1995).

[16] W. E. Wallace, J. H. van Zanten, and W. Wu, Phys. Rev. E 52, R3329 (1995).

[17] J. H. van Zanten, W. E. Wallace, and W. Wu, Phys. Rev. E 53, R2053 (1996).

[18] K. Fukao and Y. Miyamoto, Phys. Rev. E 61, 1743 (2000).

[19] G. B. DeMaggio, W. E. Frieze, D. W. Gidley, M. Zhu, H. A. Hristov, and A. F. Yee, Phys. Rev. Lett. 78, 1524 (1997).

[20] W. J. Orts, J. H. v. Zanten, W. Wu, and S. K. Satija, Phys. Rev. Lett. 71, 867 (1993).

[21] T. Kanaya, T. Miyazaki, H. Watanabe, K. Nishida, H. Yamano, S. Tasaki, and D. B. Bucknall, Polymer 44, 3769 (2003).

[22] T. Miyazaki, K. Nishida, and T. Kanaya, Phys. Rev. E 69, 022801 (2004).

[23] O. Kahle, U. Wielsch, H. Metzner, J. Bauer, C. Uhlig, and C. Zawatzki, Thin Solid Films 313/314, 803 (1998).

[24] T. Miyazaki, A. Shimazu, T. Matsushita, and K. Ikeda, J. Appl. Polym. Sci. 78, 1818 (2000).

[25] T. Miyazaki, A. Shimazu, and K. Ikeda, Polymer 41, 8167 (2000).

[26] H. Watanabe, T. Sakamoto, and T. Kotaka, Macromolecules
18, 1436 (1985).

[27] K. Dalnoki-Veress, J. A. Forrest, C. Murray, C. Gigault, and J. R. Dutcher, Phys. Rev. E 63, 031801 (2001).

[28] See Fig. 1 in Ref. [10].

[29] T. G. Fox and P. J. Flory, J. Appl. Phys. 21, 581 (1950).

[30] J. A. Forrest and K. Dalnoki-Veress, Adv. Colloid Interface Sci. 94, 167 (2001).

[31] K. Fukao and Y. Miyamoto, Phys. Rev. E 64, 011803 (2001).

[32] P. G. de Gennes, Eur. Phys. J. E 2, 201 (2000).

[33] H. Fischer, Macromolecules 35, 3592 (2002).

[34] N. Satomi, A. Takahara, and T. Kajiyama, Macromolecules 32, 4474 (1999).

[35] D. Kawaguchi, K. Tanaka, A. Takahara, and T. Kajiyama, Macromolecules 14, 6164 (2001).

[36] W. Wu, J. H. v. Zanten, and W. J. Orts, Macromolecules 28, 771 (1995).

[37] C. L. Soles, J. F. Douglas, W. Wu, and R. M. Dimeo, Phys. Rev. Lett. 88, 037401 (2002).

[38] R. Inoue, T. Kanaya, H. Yamano, K. Nishida, I. Tsukushi, and K. Shibata, in Proceedings of Slow Dynamics in Complex Systems, Sendai, 2003, edited by M. Tokuyama, AIP Conf. Proc. No. 708 (AIP, Melville, NY, 2004), p. 197. 